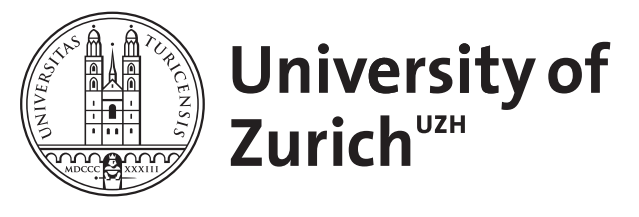

\title{
The treatment of Parkinson's disease
}

\author{
Baumann, Christian R ; Waldvogel, Daniel
}

\begin{abstract}
Parkinson's disease belongs to the most prevalent neurodegenerative disorders and manifests both with motor and non-motor symptoms. Symptomatic treatment of this disorder became more multifaceted over the past years: besides classical dopaminergic drugs and physiotherapy, novel invasive escalation treatment strategies became gold standard in many countries. On the other hand, non-motor symptoms significantly impacts quality of life in many patients which necessitates initiation of adequate therapy.
\end{abstract}

DOI: https://doi.org/10.1024/1661-8157/a001505

Posted at the Zurich Open Repository and Archive, University of Zurich ZORA URL: https://doi.org/10.5167/uzh-100412

Journal Article

Accepted Version

Originally published at:

Baumann, Christian R; Waldvogel, Daniel (2013). The treatment of Parkinson's disease. Praxis, 102(25):1529-1535.

DOI: https://doi.org/10.1024/1661-8157/a001505 
Zürich, 11. Mai 2013

\section{Die Therapie der Parkinson-Erkrankung}

\section{The Treatment of Parkinson's Disease}

Christian R. Baumann, Daniel Waldvogel

Klinik für Neurologie, UniversitätsSpital Zürich, 8091 Zürich

\section{Korrespondenzadresse}

PD Dr. med. Christian Baumann

Klinik für Neurologie

UniversitätsSpital Zürich

Frauenklinikstrasse 26

8091 Zürich

Tel $\quad+41442555503$

Fax +41442554380

Email christian.baumann@usz.ch

Anzahl Zeichen (Titel) 37

Anzahl Zeichen (Text) 15678

Anzahl Figuren 2

Anzahl Tabellen 2 


\section{Zusammenfassung}

Die Parkinson-Erkrankung gehört zu den häufigsten neurodegenerativen Erkrankungen und umfasst sowohl motorische als auch nicht-motorische Symptome. Die symptomatische Therapie dieser Erkrankung ist in den letzten Jahren vielfältiger geworden: Nebst den klassischen medikamentösen Therapiestrategien, die vorwiegend das dopaminerge System beeinflussen, und der Physiotherapie für die motorischen Symptome stehen heute in vielen Ländern zusätzlich operative Eskalationstherapien zur Verfügung. Andererseits beeinträchtigen die nicht-motorischen Symptome der Erkrankung viele Patienten signifikant und bedürfen ebenfalls einer adäquaten Behandlung.

\section{Summary}

Parkinson's disease belongs to the most prevalent neurodegenerative disorders and manifests both with motor and non-motor symptoms. Symptomatic treatment of this disorder became more multifaceted over the past years: Besides classical dopaminergic drugs and physiotherapy, novel invasive escalation treatment strategies became gold standard in many countries. On the other hand, non-motor symptoms significantly impacts quality of life in many patients which necessitates initiation of adequate therapy.

\section{Resumé}

La maladie de Parkinson est une maladie neurologique chronique neurodégénérative fréquente et présente avec des troubles moteurs et non-moteurs progressive. Récemment, le traitement de cette maladie est devenu plus varié. Ainsi que l'usage des médicaments dopaminergique et de la physiothérapie, beaucoup des centres offrent des nouveaux traitements d'escalade. En outre, les troubles non-moteurs détériorent da qualité de vie de beaucoup des patients et nécessitent l'initiation d'un traitement adéquat.

\section{Schlüsselwörter}

Parkinson-Erkrankung, Dopamin, Levodopa, Dopaminagonisten

Parkinson's disease, dopamine, Levodopa, dopamine agonists

Maladie de Parkinson, dopamine, Levodopa, dopamine agonists 


\section{Die Parkinson-Erkrankung - eine Systemerkrankung}

Die Parkinson-Erkrankung (synonym: der Morbus Parkinson) ist eine der häufigsten neurologischen Erkrankungen, deren Symptome sich aber keineswegs auf motorische Auffälligkeiten beschränken. Dies hängt damit zusammen, dass die neurodegenerativen Prozesse der Parkinson-Erkrankung weit umfassender sind als eine blosse pathologische Ablagerung von alpha-Synuclein in der Substantia nigra pars compacta. Diese falsch gefalteten Proteine finden sich nahezu ubiquitär im zentralen, aber auch im autonomen Nervensystem [1,2]. Zudem finden sich auch Zeichen anderer ProteinaggregationsErkrankungen wie zum Beispiel die Ablagerung von Tau und Amyloid-beta, die ihrerseits eher typisch für die Alzheimer-Erkrankung sind [3]. In den letzten Jahren sind die nichtmotorischen Symptome der Erkrankung sowohl klinisch als auch wissenschaftlich immer mehr in den Vordergrund getreten. Dies ist folgerichtig, zumal viele Parkinson-Patienten mehr unter nicht-motorischen Symptomen als unter den „klassischen“ motorischen Beschwerden leiden. Die vorliegende Übersichtsarbeit schliesst daher die Behandlung der nicht-motorischen Symptome mit ein - wenngleich die wissenschaftliche Evidenz für die Behandlung vieler Symptome weiterhin ungenügend ist.

\section{Diagnose}

Vor Einleitung einer Behandlung ist wie immer eine korrekte Diagnosestellung erforderlich. Für die Diagnose einer Parkinson-Erkrankung sind mindestens eine Bradykinesie (verlangsamte Bewegungen bzw. verminderter Bewegungsumfang) sowie mindestens ein weiteres der folgenden Symptome notwendig: Rigor, Tremor, posturale Instabilität, wobei letztere meist erst im Verlauf auftritt. Die Krankheit beginnt mit einseitiger Betonung dieser extrapyramidalen Symptome. Zeichen wie fehlende Asymmetrie, frühe Stürze, frühe autonome Funktionsstörungen, frühe dementielle Entwicklung, positive Pyramidenbahnzeichen, eine ausgeprägte Betonung der extrapyramidalen Symptome in den Beinen, Myokloni, sensomotorische Ausfälle, eine Ataxie oder Augenmotorikstörungen müssen zwingend an eine andere Diagnose denken lassen (eine Übersicht hierzu findet sich hier: www.evidentia.ch). Rigor und Akinesie müssen beim Morbus Parkinson auf Levodopa ansprechen.

Bereits bei der Diagnosestellung sollten die nicht-motorischen Symptome erfragt und gegebenenfalls abgeklärt werden. 


\section{Behandlung der motorischen Symptome}

Ziel der medikamentösen und chirurgischen Behandlung ist die Rekonstitution einer möglichst normalen Netzwerk-Funktion der Basalganglien, welche durch den zunehmenden Wegfall von Dopamin-produzierenden Zellen in der Substantia nigra pars compacta ein verändertes Output-Signal zurück an den Kortex senden. Daneben sollte aber nicht vergessen werden, dass gewisse Symptome wie die posturale Instabilität und die Gangstörung weder der medikamentösen noch der chirurgischen Behandlung zugänglich sind. Nicht zuletzt aus diesem Grund ist für die Mehrheit der Parkinson-Patienten die Einleitung einer Physiotherapie mit insbesondere Stand-, Gang- und Gleichgewichtstraining sinnvoll. Neuere Studien weisen darauf hin, dass auch alternative Verfahren wie Tai Chi oder Tango-Tanzen einen positiven Einfluss auf gewisse motorische Funktionen haben können $[4,5]$.

\subsection{Levodopa}

Levodopa (oder L-dopa) ist seit den 60er-Jahren und bis heute eine der tragenden Säulen der Parkinson-Behandlung. Es ist die biochemische Vorstufe von Dopamin und im Gegensatz zu Dopamin, welches nicht Blut-Hirn-Schranken-gängig ist, als orale Therapie geeignet. Da es im Blut rasch enzymatisch metabolisiert wird, ist in jedem LevodopaPräparat ein Enzymhemmer (Dopa-Decarboxylase-Hemmer: Carbidopa oder Benserazid) enthalten. Die Dosisangaben der einzelnen Präparate ist daher oft etwas verwirrlich: umgangssprachlich wird beispielsweise häufig von 250mg-Präparaten gesprochen, wenngleich der Levodopa-Anteil nur 200mg ist. Dies ist insbesondere dann zu berücksichtigen, wenn von einem Präparat auf ein anderes umgestellt wird.

Beim dreifachen Kombinationspräparat Stalevo ${ }^{\circledR}$ kommt noch Entacapon hinzu, ein Hemmer der Catechol-O-Methyltransferase (COMT). Durch diese zusätzliche Hemmung eines peripheren Abbauweges von Levodopa kommt es zu einer höheren und leicht verlängerten Bioverfügbarkeit von Levodopa [6]. Entacapon ist daneben als Einzelmedikation erhältlich, wie auch der andere COMT-Hemmer Tolcapon. Dieser wirkt zusätzlich zentral, hat aber bei einigen Patienten zu Leberversagen geführt und ist daher nur als Zweitlinientherapie zugelassen [7]. Beide Präparate können zu Diarrhoe führen. Ausserdem sollten die Patienten vorgewarnt werden, dass sich der Urin unter der Therapie rötlich verfärben kann.

Bis heute ist Levodopa das am besten wirksame medikamentöse Präparat, welches überdies in der Regel gut vertragen wird. Die häufigste Nebenwirkung, Nausea, ist bei den meisten 
Patienten nur passager und kann durch die Zugabe von Domperidon (Motilium ${ }^{\circledR}$ ) zu Beginn der Behandlung meist gut kontrolliert werden. Das Problem der Behandlung mit Levodopa sind Langzeitkomplikationen: mehr oder weniger dosisabhängig kommt es im Verlauf zu motorischen Fluktuationen. Zu Beginn der Behandlung hält die optimale Wirkung der Substanz über den ganzen Tag an, die Patienten erleben einen sogenannten „honey-moon“. Im Verlauf spüren die Patienten jedoch immer schneller eine Wirkabnahme (ein „wearing off"), welche zu Blockaden führt. Beim Erreichen von Spitzenspiegeln im Blut treten hingegen zunehmend unwillkürliche Überbewegungen (,peak dose“-Dyskinesien) auf, so dass nach Jahren unter Behandlung einzelne Patienten zwischen „off“ und „on“ mit Dyskinesien hin und her wechseln (Abbildung 1) [6]. Aus diesem Grund wird gelegentlich bei jüngeren Patienten (biologisch unter 70 Jahre) ohne die Lebenserwartung beeinträchtigende Komorbiditäten die Behandlung mit Dopamin-Agonisten statt mit Levodopa begonnen, wobei die DopaminAgonisten wie unten aufgeführt nebenwirkungsreicher sind.

Eine Übersicht über die aktuell verfügbaren Levodopa-Präparate und die entsprechenden Dosierungen findet sich in Tabelle 2.

\subsection{Dopamin-Agonisten}

Die wichtigste Alternative zu Levodopa stellen die Dopamin-Agonisten dar, welche strukturell komplett verschieden von Dopamin sind, jedoch an denselben postsynaptischen striatalen Dopaminrezeptoren andocken (Tabelle 2). Es gibt verschiedene Untertypen von Dopaminrezeptoren, welche die verschiedenen Dopamin-Agonisten mit unterschiedlicher Affinität ansteuern. Strukturell zählen Ropinirol, Pramipexol und Rotigotin zu den Nicht-ErgotDerivaten, während Cabergolin ein Ergot-Derivat ist. Letzteres hat den Nachteil, dass es zu kardialen und retroperitonealen Fibrosen führen kann und deshalb nur in zweiter Linie eingesetzt werden sollte. Ein anderes potentes Ergot-Derivat, Pergolid, ist aus diesem Grund vom Markt genommen worden. Zu beachten ist ferner, dass die Halbwertszeiten zwar stets länger als jene von Levodopa, unter den Präparaten jedoch deutlich unterschiedlich sind. Da grundsätzlich eine möglichst kontinuierliche Stimulation der Dopaminrezeptoren angestrebt wird, sollten man primär eine langwirksame Behandlung mit retardiertem Pramipexol oder Ropinirol oder aber eine transdermale Behandlung mit dem Rotigotin-Patch versuchen. Die kontinuierliche Stimulation ist nicht nur der Compliance der Patienten zuträglich, sondern wahrscheinlich auch assoziiert mit einem verminderten Auftreten von postsynaptischen Adaptationsmechanismen und Medikations-bedingten Dyskinesien, welche bei DopaminAgonisten seltener auftreten als unter Levodopa [8]. 
Daraus leiten sich die Vorteile der Therapie mit Dopamin-Agonisten ab: die längere Wirkdauer und das deutlich reduzierte Auftreten von motorischen Komplikationen ist der Grund, weshalb viele Neurologen bei jungen oder ansonsten gesunden Patienten mit langer Lebenserwartung zu dieser Strategie anstelle von Levodopa greifen. Die Therapie mit Dopamin-Agonisten ist jedoch nicht unproblematisch. Dopamin-Agonisten sind deutlich teurer als Levodopa und haben mannigfaltige Nebenwirkungen, neben der fast obligaten Nausea bei Therapiebeginn auch Beinödeme, Tagesschläfrigkeit mit Schlafattacken und Halluzinationen. Insbesondere auf Impuls-Kontroll-Störungen sollte ein Augenmerk gerichtet werden: vielen Patienten entwickeln Hypersexualität, Spielsucht, Kaufsucht, Fress-Sucht oder andere Verhaltensauffälligkeiten unter dieser Therapie, was zu erheblichen psychosozialen und dramatischen legalen Konsequenzen führen kann [9].

Bei vielen Patienten werden im Krankheitsverlauf Dopamin-Agonisten und Levodopa kombiniert, wobei auch noch die nachfolgend genannten Präparate hinzugefügt werden können.

\subsection{Weitere orale dopaminerge Therapiestrategien}

Als wichtiges weiteres Standbein haben sich die Hemmer der Monoaminooxidase B (MAOB) etabliert. Insbesondere Rasagilin (Azilect $\left.{ }^{\circledR}\right)$ wird aufgrund seines günstigen Nebenwirkungsprofils und der einfachen Dosierung (1x1mg pro Tag) häufig eingesetzt, sowohl in der Behandlung des frühen als auch des späten Parkinsons. Die in der Wirkung dem Levodopa und den Dopamin-Agonisten unterlegene Substanz führt zur Hemmung eines Abbauprozesses von Dopamin und damit zu einer Wirkverstärkung. Bei der Gabe von anderen MAO-Hemmern, Fluoxetin, Fluvoxamin oder Pethidin sollte jedoch auf diese Behandlung verzichtet werden. Aufgrund neuerer Studienergebnisse ist es ausserdem nicht ausgeschlossen, dass der Einsatz von Rasagilin den Krankheitsverlauf günstig beeinflusst, die Evidenz dazu ist aber umstritten [10]. Ebenfalls sowohl als Mono- als auch in Kombinationstherapie kann der ältere MAO-B-Hemmer, Selegilin, verschrieben werden. Bei dieser Substanz gibt es zahlreiche Kontraindikationen (medikamentös, Krankheiten), die vor Einleitung der Behandlung beachtet werden müssen.

Amantadin, ein Antagonist der N-Methyl-D-Aspartat- (NMDA-) Rezeptoren, ist ein ursprünglich gegen Grippe eingesetztes Medikament, welches bei Parkinson-Patienten einen günstigen Effekt gezeigt hat und bis heute in der Parkinson-Therapie Verwendung findet. Der genaue Wirkmechanismus ist nicht bekannt. Amantadin hat einerseits eine anticholinerge Wirkung und beeinflusst andererseits die NMDA-Rezeptoren, welche bei Bindung das Glutamat-System aktivieren. Orales Amantadin (Ziel: 3x100mg) wirkt 
symptomatisch gegen motorische Parkinson-Symptome wie auch gegen Levodopainduzierte Dyskinesien, wobei es häufig nach wenigen Monaten zu einem Wirkverlust kommt. Daneben wird intravenöses Amantadin (3x100mg) angewandt bei ausgeprägten Parkinson-Symptomen mit der Unmöglichkeit oraler Aufnahme wie zum Beispiel bei postoperativen Patienten. Vor Therapiebeginn sollten mittels EKG ein AV-Block Grad II/III oder QT-Pathologien ausgeschlossen werden, ausserdem sind unter der Behandlung nicht selten psychotische Symptome zu beobachten.

\subsection{Therapie-Eskalationen}

Wenn vor wenigen Jahren therapeutische Eskalationen noch einer verschwindend kleinen Minderheit von Parkinson-Patienten vorbehalten waren, so haben sie sich inzwischen zu einem weiteren Goldstandard in der Behandlung entwickelt. Klassischerweise wird die Therapie-Eskalation dann in Betracht gezogen, wenn die motorischen Symptome durch orale Behandlungsstrategien nicht mehr genügend kontrolliert werden können, also vor allem bei therapierefraktärem Parkinson-Tremor oder bei motorischen Fluktuationen. Vor allem die tiefe Hirnstimulation ist zu einem wichtigen Pfeiler in der Parkinson-Behandlung geworden. Weltweit sind schätzungsweise über 100‘000 Patienten mit diesem Verfahren behandelt worden. In den letzten Jahren und unter dem Eindruck ausgezeichneter Therapie-

Ergebnisse bei früherer Anwendung rückt man nach und nach von der Haltung ab, mit der Therapie-Eskalation so lange als möglich zuzuwarten. Eine kürzlich publizierte europäische Studie belegte den Vorteil einer früheren Indikationsstellung der tiefen Hirnstimulation [11].

Bei der tiefen Hirnstimulation werden bilateral Elektroden stereotaktisch in bestimmte Hirnareale implantiert. Diese Elektroden sind an der Schädel-Kalotte befestigt und mittels subkutan verlaufenden Kabels mit dem eigentlichen Stimulator verbunden, der ebenfalls subkutan implantiert wird (Abbildung 2). Der Stimulator kann von aussen mittels Induktion sowohl gelesen als auch programmiert werden, was therapeutische Anpassungen jederzeit erlaubt, sowohl durch den Arzt als auch innerhalb festgelegter Grenzen durch den Patienten. Bei Parkinson-Patienten werden die Elektrodenspitzen meist im Nucleus subthalamicus oder im Globus pallidus pars interna implantiert, bei Tremor-dominanten Patienten ausnahmsweise auch im Nucleus ventralis intermedius des Thalamus. Durch die hochfrequente und in der Regel inhibierende Pulsabgabe, deren physiologischer Effekt nicht vollständig verstanden ist, kommt es zu einer Besserung der motorischen Symptome, und je nach gewähltem Zielgebiet zu einer Minderung der motorischen Fluktuationen, weniger Dyskinesien und einer partiellen Einsparung der oralen Medikation. Ziel ist die Erhöhung der Selbständigkeit und der Lebensqualität. 
Auch die Stimulation kann Nebenwirkungen bedingen. Nebst den relativ geringen Operationsrisiken und den Hardware-bedingten Nebenwirkungen können je nach Elektrodenlage und Stärke der Stimulation benachbarte Strukturen mitmoduliert werden. Dies kann beispielsweise zu Dysarthrie, aber auch zu submanischem Zustand oder anderen Verhaltensstörungen führen, welche durch sorgfältige Applikation jedoch in der Regel minim gehalten oder vermieden werden können. Daher bedingt diese Therapie sowohl operativ als auch bezüglich der neurologischen Betreuung eine hohe Erfahrung und wird nur an wenigen Zentren in der Schweiz angeboten. Die Therapie ist aufwendig und teuer. Wichtige Kontraindikationen sind hohes Alter, Demenz, Depression, chirurgische Kontraindikationen sowie fehlendes deutliches Ansprechen auf Levodopa.

Als weitere Therapie-Eskalation müssen die Pumpensysteme genannt werden: die subkutane Apomorphin-Pumpe und die duodenale bzw. jejunale Duodopa ${ }^{\circledR}$-Pumpe. Apomorphin ist ein potenter Dopaminagonist mit raschem Wirkverlust, welcher über ein Pumpensystem kontinuierlich subkutan verabreicht werden kann. Duodopa ${ }^{\circledR}$ ist in ein Gel eingebettetes Levodopa-Präparat, welches ebenfalls mittels externer Pumpe kontinuierlich in den Dünndarm appliziert wird. Beide Systeme führen zu einer kontinuierlichen Stimulation der Dopamin-Rezeptoren und somit zu einer Regredienz oder Remission der motorischen Fluktuationen. Sie sind auch bei Patienten mit dementieller Entwicklung oder Depression anwendbar und stellen gerade für diese Patienten eine wichtige Alternative zur tiefen Hirnstimulation dar. Beide Systeme erlauben ausserdem die Gabe von Boli bei momentan schlechter Motorik. Nachteilig ist für auf Selbständigkeit bedachte Patienten das externe Pumpensystem und der relativ grosse pflegerische Aufwand (z.B. Stoma-Pflege bei Duodopa ${ }^{\circledR}$-Patienten). Bei regelmässigen subkutanen Injektionen können ApomorphinPatienten ausserdem eine schmerzhafte Verhärtung an den diversen Injektionsorten entwickeln. Beide Therapien sind teuer und technisch anspruchsvoll.

\section{Behandlung der nicht-motorischen Symptome}

Wie eingangs erwähnt, sind die nicht-motorischen Symptome bei Parkinson-Patienten häufig und nicht selten bezüglich der Lebensqualität sogar im Vordergrund. Eine sorgfältige Anamnese mit gezielter Suche von nicht-motorischen Symptomen und gegebenenfalls Einleitung einer geeigneten Therapie ist daher wichtig. Insgesamt ist die Evidenzlage für die Behandlung der nicht-motorischen Symptome deutlich geringer als für die motorischen Beschwerden. Tabelle 2 gibt eine Übersicht über wichtige nicht-motorische Symptome und deren Behandlung. 


\section{Key Messages}

1. Levodopa bleibt die wirksamste medikamentöse Behandlung von motorischen Parkinson-Symptomen, kann aber im Verlauf zu motorischen Fluktuationen führen.

2. Dopamin-Agonisten haben viel seltener motorische Spätkomplikationen, sind jedoch deutlich nebenwirkungsanfälliger und weniger wirksam als Levodopa.

3. Die tiefe Hirnstimulation ist heute standardmässige Eskalationstherapie, mit welcher nicht zu lange zugewartet werden sollte.

4. Die mannigfaltigen nicht-motorischen Symptome der Parkinson-Erkrankung müssen unbedingt erfragt und im Bedarf behandelt werden.

\section{Lernfragen}

COMT-Hemmer haben folgende Eigenschaft nicht:

1. Sie führen zu einer vermehrten Bioverfügbarkeit von Levodopa.

2. Sie führen zu einer vermehrten Bioverfügbarkeit von Dopamin-Agonisten.

3. Sie führen oft zu Diarrhoe.

4. Sie sind als Einzelmedikation und teilweise auch in Kombinationspräparaten erhältlich.

Antwort: B

Die tiefe Hirnstimulation ist erfolgreich bei folgenden Indikationen:

1. Parkinson-Patienten mit ausgeprägter Standunsicherheit.

2. Parkinson-Patienten mit dementieller Entwicklung.

3. Parkinson-Patienten mit schwerer Depression.

4. Parkinson-Patienten mit motorischen Fluktuationen.

Antwort: D 


\section{Referenzen}

1 Probst A, Bloch A, Tolnay M. New insights into the pathology of Parkinson's disease: does the peripheral autonomic system become central? Eur J Neurol 2008; 15 Suppl 1:1-4.

2 Braak H, Del Tredici K, Rüb U, de Vos RA, Jansen Steur EN, Braak E. Staging of brain pathology related to sporadic Parkinson's disease. Neurobiol Aging 2003; 24: 197-211.

3 Irwin DJ, White MT, Toledo JB, Xie SX, Robinson JL, Van Deerlin V, et al. Neuropathologic substrates of Parkinson disease dementia. Ann Neurol 2012 (Epub ahead of print)

4 Li F, Harmer P, Fitzgerald K, Eckstrom E, Stock R, Galver J, et al. Tai chi and postural stability in patients with Parkinson's disease. N Engl J Med 2012; 366: 511-9.

5 Foster ER, Golden L, Duncan RP, Earhart GM. Community-Based Argentine Tango Dance Program Is Associated With Increased Activity Participation Among Individuals With Parkinson's Disease. Arch Phys Med Rehabil 2012 (Epub ahead of print)

6 Olanow CW, Obeso JA, Stocchi F. Drug insight: Continuous dopaminergic stimulation in the treatment of Parkinson's disease. Nat Clin Pract Neurol 2006; 2: 382-92.

7 Olanow CW, Watkins PB. Tolcapone: an efficacy and safety review (2007). Clin Neuropharmacol 2007; 30: 287-94.

8 Stocchi F. The therapeutic concept of continuous dopaminergic stimulation (CDS) in the treatment of Parkinson's disease. Parkinsonism Relat Disord 2009; 15: S68-71.

9 Voon V, Gao J, Brezing C, Symmonds M, Ekanayake V, Fernandez H, et al. Dopamine agonists and risk: impulse control disorders in Parkinson's disease. Brain 2011; 134(Pt 5): $1438-46$.

10 Olanow CW, Rascol O, Hauser R, Feigin PD, Jankovic J, Lang A, et al; ADAGIO Study Investigators. A double-blind, delayed-start trial of rasagiline in Parkinson's disease. $\mathrm{N}$ Engl J Med 2009; 361: 1268-78.

11 Schuepbach WM, Rau J, Knudsen K, Volkmann J, Krack P, Timmermann L, Hälbig TD, Hesekamp H, Navarro SM, Meier N, Falk D, Mehdorn M, Paschen S, Maarouf M, Barbe MT, Fink GR, Kupsch A, Gruber D, Schneider GH, Seigneuret E, Kistner A, Chaynes P, Ory-Magne F, Brefel Courbon C, Vesper J, Schnitzler A, Wojtecki L, Houeto JL, Bataille B, Maltête D, Damier P, Raoul S, Sixel-Doering F, Hellwig D, Gharabaghi A, Krüger R, Pinsker MO, Amtage F, Régis JM, Witjas T, Thobois S, Mertens P, Kloss M, Hartmann A, Oertel WH, Post B, Speelman H, Agid Y, SchadeBrittinger C, Deuschl G; EARLYSTIM Study Group. Neurostimulation for Parkinson's disease with early motor complications. N Engl J Med 2013; 368: 610-22. 
12 Emre M, Aarsland D, Albanese A, Byrne EJ, Deuschl G, De Deyn PP, et al. Rivastigmine for dementia associated with Parkinson's disease. N Engl J Med 2004; 351: 2509-18.

13 Richard IH, McDermott MP, Kurlan R, Lyness JM, Como PG, Pearson N, et al; SADPD Study Group. A randomized, double-blind, placebo-controlled trial of antidepressants in Parkinson disease. Neurology 2012; 78: 1229-36.

Abbildung 1. Klassischer Verlauf der Parkinson-Erkrankung unter Levodopa-Behandlung. Das Schema zeigt die Verengung des therapeutischen Fensters über die Zeit auf (von A nach C). Zu Beginn ist der Patient über den Tag gut behandelt (,honey-moon“, A), im Verlauf treten „on“- (Dyskinesien) und „off“- (Blockaden, Bradykinesien) Phasen erstmals auf (B) und führen schliesslich zu erheblichen motorischen Fluktuationen, wobei der Patient nur noch während kurzer Zeitfenster gut beweglich und nicht dyskinetisch ist (C). Adaptiert nach: [6].

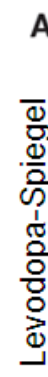

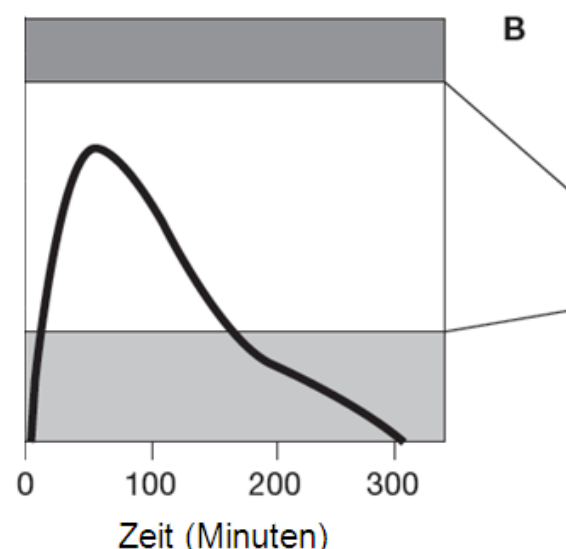

Zeit (Minuten)

B

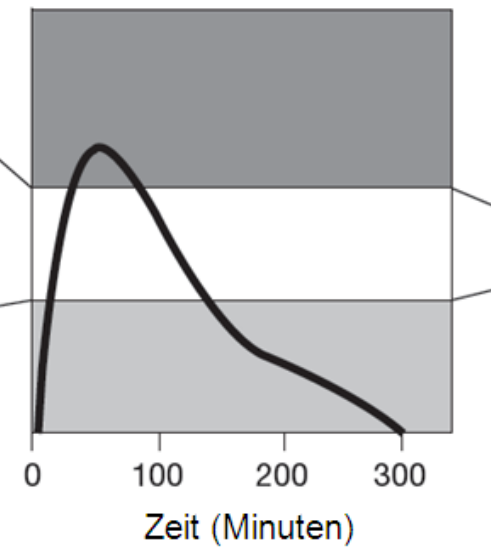

Gute Motorik («on»)
C

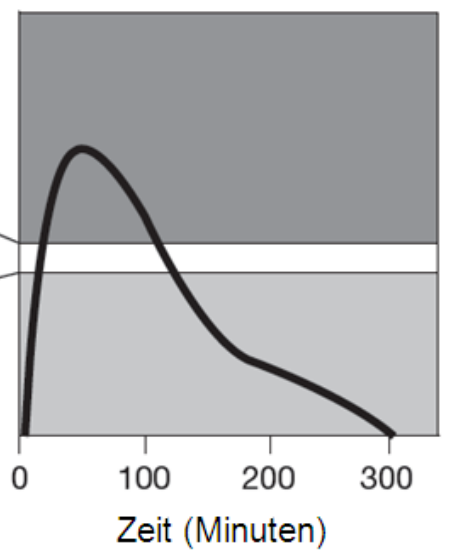

Akinetisch/blockiert («off») 

Abbildung 2. Schematische Darstellung der Anlage eines Systems zur tiefen Hirnstimulation (THS). Aus: www.evidentia.ch.

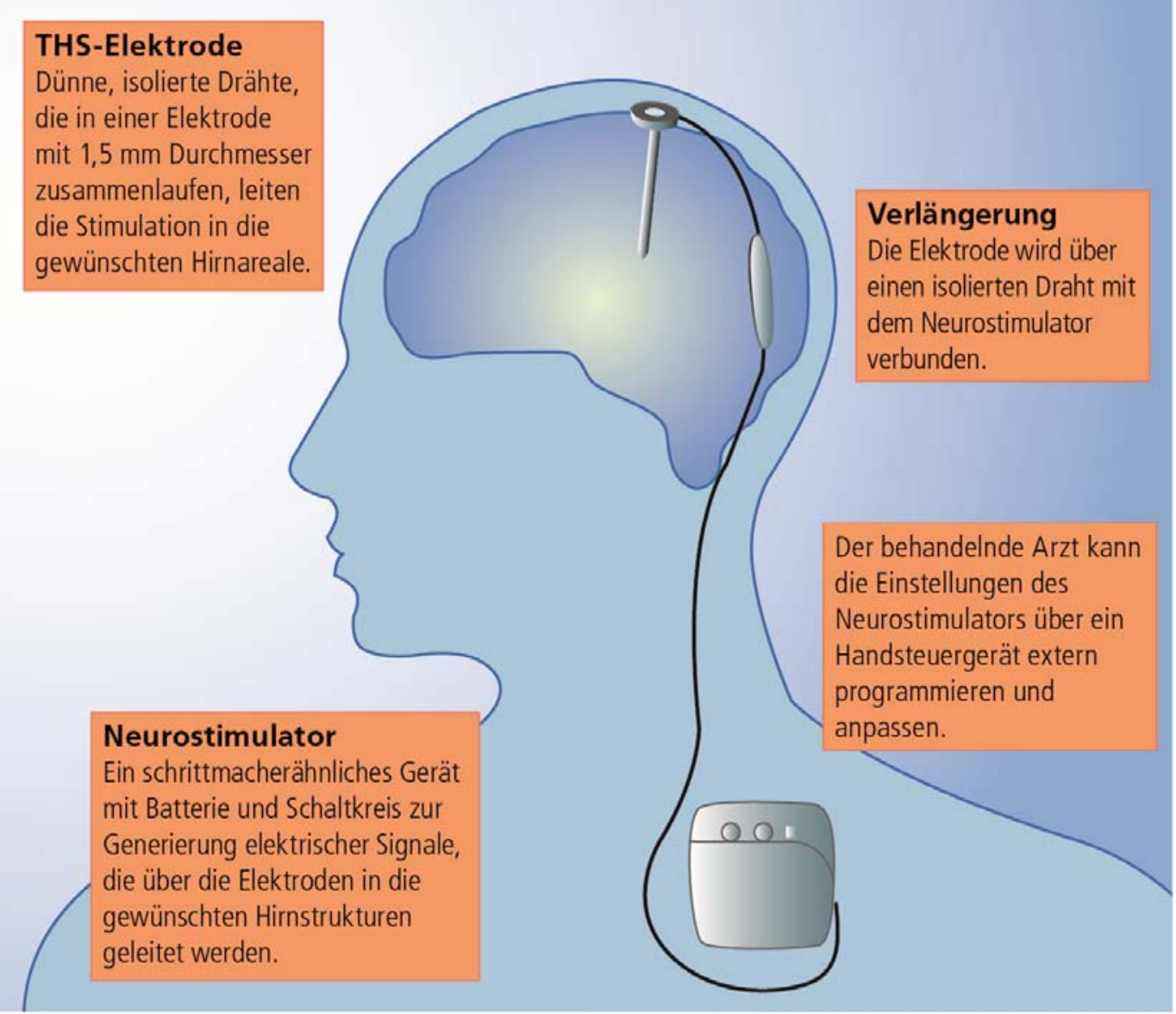


Tabelle 1. Medikamentöse Behandlung der motorischen Symptome: Übersicht über gebräuchliche dopaminerge Substanzen.

Wirkstoff Handelsname Beginn Übliche Zieldosis

\section{Levodopa-Präparate}

Levodopa/Benserazid

Levodopa/Carbidopa

$$
\begin{aligned}
& \text { Madopar }{ }^{\circledR} \\
& \text { Sinemet }{ }^{\circledR} \\
& \text { Carbidopa/ } \\
& \text { Levodopa }
\end{aligned}
$$

$3 \times 50 \mathrm{mg}$

$300-600 \mathrm{mg}$

(Levodopa)

(Levodopa)

Levodopa/Carbidopa/

Stalevo ${ }^{\circledR}$

300-600mg

Entacapon

(Levodopa)

\section{COMT-Hemmer}

Entacapon

Comtan ${ }^{\circledR}$

1x mit jeder Levodopa-Einnahme

Tolcapon

Tasmar $^{\circledR}$

$3 \times 100 \mathrm{mg}$

$3 \times 100 \mathrm{mg}$

\section{Dopamin-Agonisten}

Pramipexol

Ropinirol

Rotigotin

Cabergolin

\begin{tabular}{|c|c|c|}
\hline u.a. Sifrol ${ }^{\circledR}$ & $3 \times 0.125 \mathrm{mg}$ & $1.5-4.5 \mathrm{mg}$ \\
\hline Sifrol ER ${ }^{\circledR}$ & $1 \times 0.375 \mathrm{mg}$ & $1.5 .4 .5 \mathrm{mg}$ \\
\hline u.a. Requip ${ }^{\circledR}$ & $3 \times 0.25 \mathrm{mg}$ & $3-9(-24) \mathrm{mg}$ \\
\hline Requip & $1 \times 2 \mathrm{mg}$ & $4-10(-24) \mathrm{mg}$ \\
\hline \multicolumn{3}{|l|}{ Modutab ${ }^{\circledR}$} \\
\hline Neupro ${ }^{\circledR}$ & $1 \times 1 \mathrm{mg}$ & $8-16 \mathrm{mg}$ \\
\hline Cabaser $^{\circledR}$ & $1 \times 1 \mathrm{mg}$ & $2-3 \mathrm{mg}$ \\
\hline
\end{tabular}

\section{MAO-B-Hemmer}

Rasagilin

Selegilin

$\begin{array}{lll}\text { Azilect }^{\circledR} & 1 \times 1 \mathrm{mg} & 1 \times 1 \mathrm{mg} \\ & 5 \mathrm{mg} & \max .10 \mathrm{mg}\end{array}$


Tabelle 2. Nicht-motorische Symptome bei der Parkinson-Erkrankung (adaptiert nach: www.evidentia.ch)

\section{Autonome Symptome}

Blasenfunktionsstörungen (v.a. Urge-Inkontinenz) - Tolterodin (Detrusitol ${ }^{\circledR}$ )

- Trospiumchlorid (Spasmo-Urgenin ${ }^{\circledR}$ )

20-60mg

- Oxybutynin (z.B. Ditropan ${ }^{\circledR}$ - Restharn-Kontrolle)

$5-15 \mathrm{mg}$

Verzögerte Magenentleerung

- sakrale Neuromodulation

Obstipation

- Domperidon (Motilium ${ }^{\circledR}$ )

30-60mg

Erektile Dysfunktion

- viel Flüssigkeit und Ballaststoffe, körperliche Aktivität - Macrogol (Transipeg ${ }^{\circledR}$, Movicol ${ }^{\circledR}$ )

- ggf. Phosphodiesterase-V-Hemmer (Vardenafil,

Tadalafil, Sildenafil)

Blutdruckdysregulation, Orthostase-Probleme

- genügend Flüssigkeit, Kompressionsstrümpfe, nachts Schlafen mit angehobenem Oberkörper

- Fludrocortisol (Florinef ${ }^{\circledR}$ )

- Midodrin (Gutron ${ }^{\circledR}$ )

- Domperidon (Motilium ${ }^{\circledR}$ )

30-60mg

Sialorrhoe

- Optimierung der dopaminergen Behandlung

- lokale Botulinumtoxin-A-Injektionen

- ggf. Anticholinergika wie Biperiden (Akineton ${ }^{\circledR}$ )

Temperaturdysregulation

Gestörte Sudomotorik (Schwitzen)
- bislang keine Therapie bekannt

- bislang keine Therapie bekannt 


\section{Neuropsychiatrische Symptome}

Depression

\section{Angststörungen}

Demenz, Mild Cognitive Impairment (MCl)

Halluzinationen, Psychosen

Impuls-Kontroll-Störungen
Selektive Serotonin-Wiederaufnahme-Hemmer

- Paroxetin (Klasse-I-Evidenz, off-label) [13]

20-40mg

Serotonin-Noradrenalin-Wiederaufnahmehemmer

- Venlaflaxin (Klasse-I-Evidenz, off-label) [13]

75-150mg

Trizyklische Antidepressiva

- Amitryptilin (off-label)

$10-150 \mathrm{mg}$

Tetrazyklische Antidepressiva

- Mirtazapin (Remeron ${ }^{\circledR}$, off-label)

$15-45 \mathrm{mg}$

Dopaminagonisten (z.B. Pramipexol) wirken antidepressiv

- ggf. Serotonin-Wiederaufnahme-Hemmer (off-label),

Pregabalin (Lyrica ${ }^{\circledR}$, off-label)

- Medikation überprüfen (z.B. Anticholinergika, trizyklische

Antidepressiva reduzieren oder absetzen)

- Rivastigmin (Exelon ${ }^{\circledR}$, Klasse-I-Evidenz) [12]

- Primär Medikation prüfen (vorsichtiges Reduzieren oder Absetzen von Anticholinergika, trizyklischen Antidepressiva, Amantadin, MAO-B-Hemmern, Dopamin-Agonisten etc)

- Clozapin (Leponex ${ }^{\circledR}$ )

$6.25-50 \mathrm{mg}$

- Quetiapin (Seroquel ${ }^{\circledR}$, off-label)

$12.5-50 \mathrm{mg}$

- Reduktion oder Absetzen von Dopamin-Agonisten

- ggf. atypische Neuroleptika (Clozapin, Quetiapin) 


\section{Schlaf-Wach-Störungen}

Insomnie (Ein- und Durchschlafstörung)

\section{Exzessive Tagesschläfrigkeit}

Fatigue

REM-Schlaf-Verhaltensstörung

NREM-Parasomnien (z.B. Somnambulismus)

Restless legs-Symptome

Schlafapnoe

\section{Sensible Störungen}

Schmerzen

Dysästhesien
- langwirksame dopaminerge Behandlung zur Nacht - schlafanstossende Antidepressiva (z.B. Amitryptilin, tiefdosiertes Mirtazapin)

- Dopamin-Agonisten reduzieren

- Modafinil (Modasomil ${ }^{\circledR}$, off-label)

$100-200 \mathrm{mg}$

- keine erfolgsversprechende Therapie bekannt

- Clonazepam (Rivotril ${ }^{\circledR}$, off-label) zur Nacht

$0.25-1 \mathrm{mg}$

- hochdosiertes Melatonin (off-label) zur Nacht

- ggf. Clonazepam, SSRI's

- primär tiefdosierte Dopamin-Agonisten zur Nacht

- ggf. pneumologisch behandeln (CPAP)
- dopaminerge Behandlung optimieren

- konventionelle Schmerztherapeutika

- schmerzdistanzierende Antidepressiva

- ggf. tiefe Hirnstimulation bei doparesponsiven Schmerzen

wie bei Schmerzen 